\section{Metastasierender Brustkrebs: länger progressionsfrei mit Fulvestrant/Palbociclib}

Schon eine Interimsanalyse der PALOMA-3-Studie hatte gezeigt, dass Frauen mit metastasiertem hormonrezeptorpositvem Brustkrebs, die unter endokriner Therapie einen Progress entwickelt hatten, von der CDK4-/CDK6-Inhibition zusätzlich zur endokrinen Therapie profitieren. Nun liegen finale Daten vor.

In der PALOMA-3-Studie wurden insgesamt 521 Frauen mit Hormonrezeptor(HR)-positivem, HER2-negativem, metastasiertem Brustkrebs behandelt, deren Tumor unter endokriner Therapie fortge-

schritten war. Alle erhielten Fulvestrant

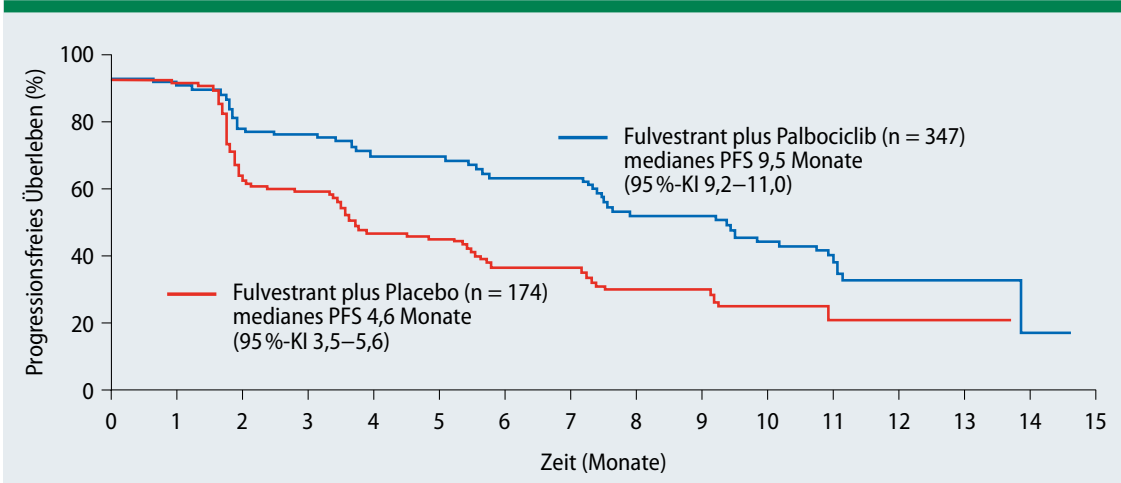

Hazard Ratio 0,46; $95 \%$-Konfidenzintervall (95\%-KI) 0,36-0,59; $<<0,0001$

Abb. 1: Kaplan-Meier-Kurven des progressionsfreien Überlebens in der Intent-to-TreatAnalyse unter Fulvestrant/Palbociclib gegenüber Fulvestrant/Placebo.

\section{Axilläre Rezidive bei Mammakarzinom: wie häufig?}

\begin{abstract}
Bei Brustkrebspatientinnen ist die Rate axillärer Rezidive nach der SentinelLymphknotenbiopsie (SLNB) normalerweise niedrig (0-2\%); nun wurden Inzidenz und Einfluss auf das Überleben in einer großen Kohorte untersucht.
\end{abstract}

R etrospektiv wurden Daten von insgesamt 14.095 Brustkrebspatientinnen (klinisch N0), die sich einer Operation und einer SLNB unterzogen hatten, betrachtet. Bei $34,5 \%$ von ihnen war eine Lymphknotendissektion erfolgt.

Nach median 55,2 Monaten wurden bei $0,51 \%$ der Patientinnen axilläre Rezidive nachgewiesen. In der multivariaten Analyse korrelierte ihr Auftreten mit einer Erkrankung vom Grad 2 oder 3, dem Fehlen einer Bestrahlung und dem Tumor-Subtyp: Die Raten betrugen bei triple-negativen Tumoren 1\%, bei sowie 2:1 randomisiert entweder den CDK4-/CDK6-Inhibitior Palbociclib oder Placebo. Primärer Endpunkt war das progressionsfreie Überleben (PFS),

das nach median 8,9 Monaten in der Palbociclib-Gruppe signifikant besser war (median 9,5 vs. 4,6 Monaten; Hazard Ratio 0,46; $\mathrm{p}<0,0001$ ) (Abb. 1). Auch das opjektive Ansprechen wurde durch die CDK4-/CDK6-Inhibition verbessert (Fulvestrant/Palbociclib: 24,6 vs. Fulvestrant/ Placebo: 10,9\%). $67 \%$ der Patientinnen erlebten unter Palbociclib einen klinischen Benefit, nur $40 \%$ unter Placebo.

Grad-3/4-Nebenwirkungen entwickelten $73 \%$ der Frauen im Fulvestrant/Palbociclib-Arm und $22 \%$ im Fulvestrant/ Placebo-Arm, am häufigsten eine Neutropenie (65 vs. $1 \%$ ).

Fazit: Fulvestrant/Palbociclib verbessert signifikant das PFS gegenüber Fulvestrant/Placebo, unabhängig vom Grad der endokrinen Resistenz, der Höhe der HRExpression und dem PIK3CA-Mutationsstatus. Die Kombination ist eine Therapieoption bei rezidivierendem HR-positivem, HER2-negativem metastasiertem Brustkrebs im Progress nach endokriner Therapie. Brigitte Schalhorn

Cristofanilli M et al. Fulvestrant plus palbociclib versus fulvestrant plus placebo for treatment of hormone-receptor-positive, HER2-negative metastatic breast cancer that progressed on previous endocrine therapy (PALOMA-3): final analysis of the multicentre, double-blind, phase 3 randomised controlled trial. Lancet Oncol. 2016;17(4):425-39

nifikant niedriger bei Patientinnen mit axillärem Rezidiv, einem Alter über 50 Jahre, lymphovaskulärer Invasion, Tumoren vom Grad 3, Makrometastasen in den Sentinel-Lymphknoten, einer Tumorgröße $>20 \mathrm{~mm}$, dem Fehlen einer Chemotherapie und bei triple-negativem Phänotyp.

HER2-positiven Tumoren 2,8\%, bei Luminal-A-Tumoren $0,4 \%$, bei HER2-negativen Luminal-B-Tumoren $0,9 \%$ und bei HER2-positiven Luminal-B-Tumoren $0,5 \%$. Anhand eines vereinfachten Scores zur Vorhersage axillärer Rezidive konnten die Patientinnen in 3 Gruppen mit signifikant unterschiedlichen Rezidivraten eingeteilt werden ( $\mathrm{p}<0,0001)$.

Das 7-Jahres-Gesamtüberleben betrug bei Patientinnen ohne axilläres Rezidiv 94,5\%, bei denen mit Rezidiv $79,9 \%$. In der multivariaten Analyse war das 5-Jahres-Gesamtüberleben sig-
Fazit: Isolierte axilläre Rezidive sind bei HER2-positiven, Hormonrezeptor-negativen, triple-negativen Tumoren häufiger und ein unabhängiger Risikofaktor für eine schlechtere Prognose, der eine systemische Therapie rechtfertigt. Allerdings muss deren Nutzen noch untersucht werden.

Judith Neumaier

Houvenaeghel $\mathrm{G}$ et al. Survival impact and predictive factors of axillary recurrence after sentinel biopsy. Eur J Cancer. 2016;58:73-82. 